\title{
Bericht über den 2.0perationskurs zur antegraden skrotalen Sklerosierung der Varicocele testis am 14. und 15.8.1992 in Hamburg
}

\section{U. Seppelt}

Diakonissen Krankenhaus, Flensburg

Nach kurzem Intervall fand bereits zum zweiten Mal wegen der Nachfrage am 15.8.1992 ein Operationskurs zur antegraden Sklerosierung der Varicocele testis im Allgemeinen Krankenhaus Barmbek, Hamburg, unter der Leitung von Herrn Prof. Dr. R. Tauber statt.

Mehr als 90 Teilnehmer aus dem gesamten Bundesgebiet, Österreich und der Schweiz nahmen teil. Die zunächst meist ungewohnten Schiffsplanken des Hamburger Museums-Schiffes Cap San Diego ließen schnell eine gemütliche Club-Atmosphäre entstehen, die geographische Distanzen schnell schrumpfen ließ. Zauberkünste eines polnischen Magiers rundeten den BegrüBungsabend ab.

Die bestechend einfache Technik der Sklerosierung nach Lokalanästhesie wurde per Video-LiveÜbertragung aus 3 Operationssälen demonstriert. Insgesamt wurden 25 Patienten operiert. Die Mehrzahl der Kursteilnehmer konnte sich durch persönliche Operations-Assistenzen von der einfachen Durchführbarkeit und der minimalen Invasivität der Methode überzeugen.

Als innovative Operations-Methode der Varicocele testis wurde auch die laparoskopische Varikozelen-Ligatur vorgestellt. Herr Rassweiler (Mannheim) demonstrierte die beeindruckende Technik ebenfalls via Video-Live-Übertragung an 2 Patienten.
Das Programm war abgerundet durch 12 Vorträge über theoretische Aspekte, Diagnostik, Therapie, Nachsorge und Spätergebnisse der Varikozele in umfassender Form.

Die abschließende Diskussion bekräftigte den Stellenwert der antegraden Sklerosierung in der Therapie der Varikozele mit einer Erfolgsrate von $94 \%$. Die Zukunft der laparoskopischen Varikozelen-Resektion scheint noch nicht absehbar, denkbare Indikationen sind Varikozelen-Persistenzen nach herkömmlichen Operationen oder Sklerosierungen. Es war insgesamt eine gelungene, mit Engagement organisierte und durchgeführte Veranstaltung.

Prof.Dr.med. U. Seppelt

Chefarzt der Urologischen Klinik

Diakonissen Krankenhaus

Marienhölzungsweg 2

2390 Flensburg 